\title{
Fabrication of elastomeric stamps with polymer-reinforced sidewalls via chemically selective vapor deposition polymerization of poly (p-xylylene)
}

\author{
Kahp Y. Suh and Robert Langer ${ }^{\text {a) }}$ \\ Department of Chemical Engineering, Massachusetts Institute of Technology, Cambridge, \\ Massachusetts 02139 \\ Jörg Lahann \\ Department of Chemical Engineering, University of Michigan, Ann Arbor, Michigan 48109
}

(Received 1 July 2003; accepted 23 September 2003)

\begin{abstract}
We report on the preparation of polydimethylsiloxane stamps with selectively grown polymer sidewalls by chemical vapor deposition polymerization of poly( $p$-xylylene). Using a thin iron layer as an inhibitor, the deposition occurs only on the sidewalls of the features in relief, resulting in a polymer-reinforced stamp. The wetting properties of stamps can be restored after removing the thin iron layer with an acidic solution, which has been verified by pattern transfer to an underlying substrate using molding and microcontact printing. (C) 2003 American Institute of Physics.
\end{abstract}

[DOI: $10.1063 / 1.1628392$ ]

Soft lithography is the collective name of microfabrication techniques that involve replication of features into elastomers, such as polydimethylsiloxane (PDMS). These techniques have a broad range of applications in materials science, chemistry, and biology. ${ }^{1}$ However, the extension of soft lithographic methods to achieve structures below 500 $\mathrm{nm}$ or, in particular, in the sub-100 $\mathrm{nm}$ range has been limited in part due to the low elastic modulus of PDMS. In addition, the high compressibility of PDMS $\left(\sim 2.0 \mathrm{~N} / \mathrm{mm}^{2}\right.$ for Sylgard 184) gives rise to deformation, buckling, or collapse of shallow relief features. ${ }^{2,3}$ High surface tension causes rounding of sharp corners when released from the master. ${ }^{4}$ These mechanical shortcomings of PDMS are currently limiting the successful replication and generation of features with high pattern fidelity in the submicron range.

To improve the performance of pattern transfer, Michel and co-workers introduced alternative siloxane polymers having improved mechanical properties compared to Sylgard 184 PDMS. ${ }^{5}$ The polymer was based on a composite of vinyl and hydrosilane end-linked chains, which has been successfully applied to high-density patterns at the $100 \mathrm{~nm}$ scale with the aid of the enhanced modulus $\left(\sim 9 \mathrm{~N} / \mathrm{mm}^{2}\right){ }^{5}$ Recently, Whitesides and co-workers ${ }^{6}$ presented a bilayer PDMS stamp that uses a thick, flexible 184 PDMS slab to support a thin, stiff PDMS underlayer. With this enforced stamp, structures with $<100 \mathrm{~nm}$ resolution were created using phase-shifting lithography. ${ }^{6}$ In addition to these 184 PDMS based studies, a photocurable PDMS $^{7}$ and polyolefin stamps $^{8}$ were recently developed for soft lithographic applications in the nanometer regime.

Here, we present a fabrication method to enhance the mechanical properties of PDMS stamps using selective deposition of thin polymer films prepared by chemical vapor deposition (CVD). This technique not only provides en-

\footnotetext{
a) Author to whom correspondence should be addressed; electronic mail: rlanger@mit.edu
}

hanced mechanical properties in comparison to Sylgard 184 PDMS, but also allows for a flexible solution to surface engineering virtually on any stamp since the deposition can be carried out regardless of the nature of the substrate..$^{9-12}$

Our method consists of three steps [Fig. 1(a)]. Initially, PDMS stamps were fabricated by casting a conventional PDMS (Sylgard 184 silicon elastomer, Dow Corning) against a complementary relief structure prepared by photolithographic method and then cured at $70{ }^{\circ} \mathrm{C}$ for $1 \mathrm{~h}$ with $10 \mathrm{wt} \%$ of the curing agent. For deposition of the inhibition layer, a 3 $\mathrm{nm}$ titanium film (Ti) was first applied on the surface as an adhesion promoter by electron-beam evaporation (Sloan Pak 8 evaporator in MTL) at a rate of $1 \AA / s$ and a vacuum pressure of $1.6 \times 10^{-6}$ (base line pressure $=1.1 \times 10^{-6}$ ). Then a thin iron film $(3-5 \mathrm{~nm})$ was deposited on the surface with a relatively fast rate $(\sim 2 \AA / s)$. In an earlier study, iron was found to prevent nucleation and chain propagation during chemical vapor polymerization, thereby allowing selective polymer deposition. ${ }^{13,14}$ During electron-beam evaporation, it is expected that the iron density would be substantially small on the inner sidewalls of the stamp compared to the flat top and bottom surfaces as long as the film is ultrathin.

The polymer films consist of poly( $p$-xylylene) (PPX) and were homogenously deposited by means of CVD polymerization. The CVD process was adapted from a procedure of Gorham that was commercially used to produce solvent and pinhole-free coatings. ${ }^{15}$ In the CVD process, the dimer [2.2]paracyclophane is transferred into a pyrolysis zone after its sublimation. Control of polymerization parameters allows selective cleavage of the $\mathrm{C}-\mathrm{C}$ single bonds resulting in the corresponding quinodimethanes. ${ }^{16,17}$ Reaction conditions must be controlled to avoid decomposition of the functional groups under the conditions of quantitative conversion into quinodimethanes. Subsequently, $p$-quinodimethanes are transferred to a cooler deposition chamber where they spontaneously polymerized.

The resulting stamps were characterized by atomic force 
(a)
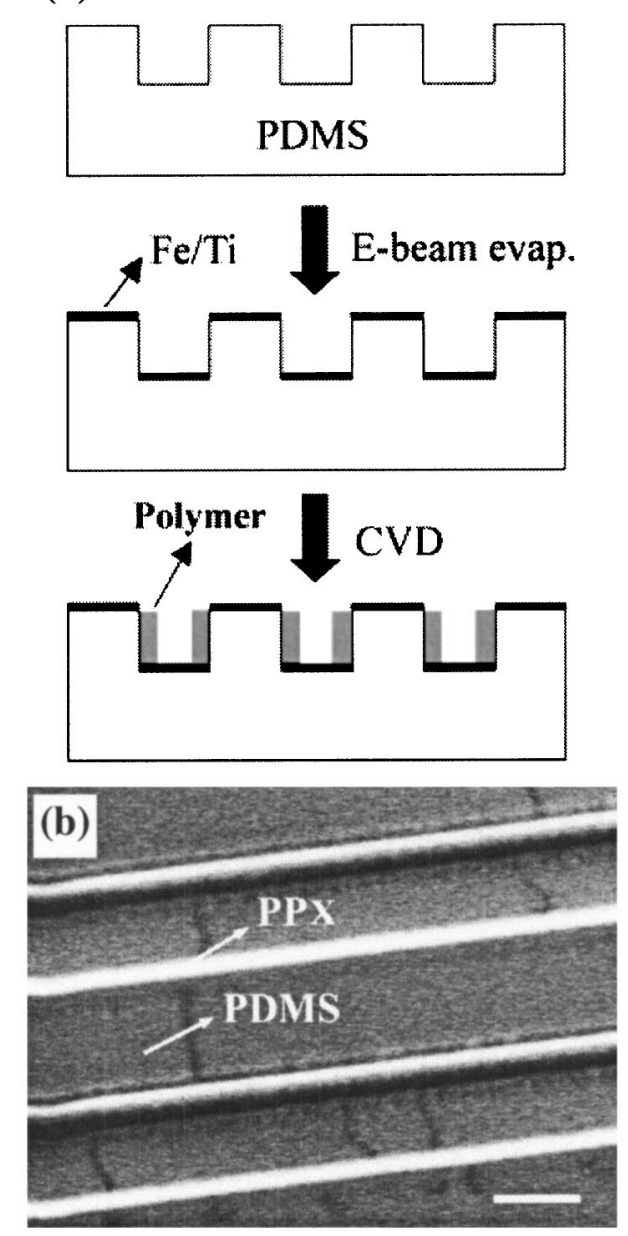

FIG. 1. (a) A schematic diagram of the experimental procedure. (b) A SEM image of a PDMS stamp with a selectively grown PPX film along the sidewall. Note the sharp difference in colors between PDMS and PPX. The lateral thickness of PPX layer is $100 \mathrm{~nm}$, which gives an increased aspect ratio of 1.9. The bar scale indicates $500 \mathrm{~nm}$.

microscopy (AFM) (Digital Instrument) in tapping mode. For high aspect ratio structures, the depths determined by AFM were smaller than expected possibly due to tip effects. Therefore, we additionally transferred the features of the stamp into a polymer with a low glass transition temperature by capillary force lithography ${ }^{18}$ to verify the success of selective deposition. For this purpose, a thin film of poly(styrene-butadiene-styrene) (SBS) (10 wt \% in toluene) was prepared by spin coating at $3000 \mathrm{rpm}$ for $20 \mathrm{~s}$ on silicon substrates. The film thickness was $1.3 \mu \mathrm{m}$ as measured by elliosometry. Prior to the stamp placement, the stamp was immersed in a $1 \mathrm{wt} \% \mathrm{HF} /$ water solution for $30 \mathrm{~s}$ to remove the metal layers. The PDMS stamp with selectively grown PPX film was then placed on the SBS surface and the temperature was raised above the glass transition temperature $\left(36^{\circ} \mathrm{C}\right)$, typically, for $5 \mathrm{~h}$ at $80^{\circ} \mathrm{C}$. The usefulness and conformal wetting of the modified PDMS stamp were further demonstrated by pattern transfer using microcontact printing $(\mu \mathrm{CP})$ onto a gold substrate followed by wet etching. Initially, the stamp was soaked with a $1 \mathrm{mM}$ solution of hexadecanethiol in ethanol. After drying, the stamp was applied onto a gold $(200 \mathrm{~nm}) / \mathrm{Ti}(5 \mathrm{~nm}) / \mathrm{Si}$ substrate for $20 \mathrm{~min}$ and then the substrate was immediately immersed in the etch solution (1 M KOH, $0.1 \mathrm{M} \mathrm{KCN}$ ) for 5 min. ${ }^{19}$ The sample
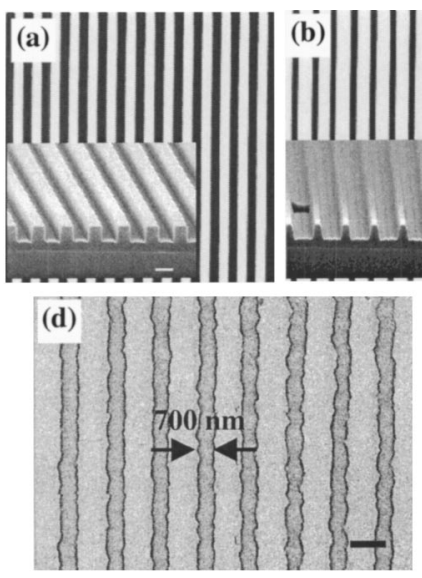
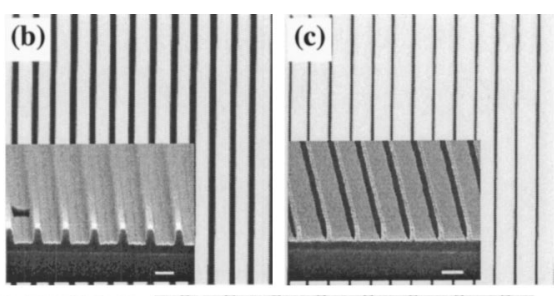

(e)

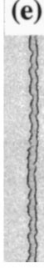

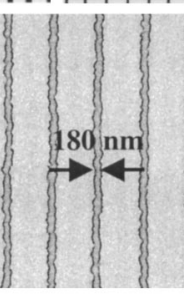

FIG. 2. (a)-(c) AFM images of PPX deposited stamps (700 nm line-andspace) along with the SEM micrographs of replicated structures of SBS: (a) a fresh stamp without polymer deposition, (b) a stamp with $100 \mathrm{~nm}$ laterally grown PPX film, and (c) a stamp with $260 \mathrm{~nm}$ laterally grown PPX film. The step height in (c) was measured to be much smaller than the expected value, which was attributed to the AFM tip effects. The scan size is $20 \times 20 \mu \mathrm{m}^{2}$. (d)-(e) SEM images of Au lines obtained by $\mu \mathrm{CP}$ onto a gold substrate followed by wet etching. The bar scales indicate $1 \mu \mathrm{m}$.

was then rinsed with distilled water and ethanol and dried in a stream of nitrogen. The resulting structures were examined by scanning electron microscopy (SEM).

Our original results with parallel channels (equal line and space width) show that Sylgard 184 PDMS starts to collapse as the width of the channels is decreased below 700 $\mathrm{nm}$ at a step height of $950 \mathrm{~nm}$. The collapse is mainly caused by hydrophobic interactions. When released from the silicon dioxide master, groups of two or three channels appear to be merged to form a larger channel, which is on a few micrometer length scale. This trend increases with decreasing line width such that stamp features were completely destroyed for channel widths below $500 \mathrm{~nm}$. In addition, at this step height the stamp is vulnerable to additional exposure to heat or solvent even after the stamp is successfully released from the master. In the PDMS stamp treated with CVD, however, no collapsing of line features was observed as the deposited polymer acts as a supporting layer to increase the overall mechanical properties. It is also noteworthy that the initial wetting properties of PDMS can be maintained regardless of the CVD process as the deposition occurs only on the inner sidewalls, not on the contacted surface.

Figure 1(b) shows a typical SEM micrograph of the PDMS surface after selective deposition of a thin PPX film $(\sim 100 \mathrm{~nm})$ on the sidewalls. Some metal cracks are seen on the bottom of the stamp, which did not significantly affect the selective deposition. The white color at the edge of the object indicates the grown PPX film (note the different colors between PDMS and PPX) and the image shows the selectively grown PPX films along the sidewalls. No deposition on either top or bottom surfaces was observed. To examine the depth profiles after selective deposition, AFM measurements were carried out for $700 \mathrm{~nm}$ line-and-space pattern with two different film thicknesses as shown in Fig. 2. In case of the reference (a PDMS stamp without polymer deposition), the initial step height turned out to be 940 $\mathrm{nm}$, which was nearly the same as the expected value (950 $\mathrm{nm})$. In case of the stamp with $100 \mathrm{~nm}$ PPX film, the line- 
width is decreased from 700 to $500 \mathrm{~nm}$ while maintaining the initial step height, which is further evidence of selective deposition. In the case of the stamp with a relatively thick film $(260 \mathrm{~nm})$, the linewidth is decreased from 700 to 180 $\mathrm{nm}$ with a step height of about $500 \mathrm{~nm}$. This depth is much smaller than the expected value, which could be attributed to the AFM tip effects resulting from the high aspect ratio or alternatively, may indicate polymer deposition in the channels. Throughout the samples, surface roughness was measured to range from 2 to $3 \mathrm{~nm}$, further indicating selective deposition.

To verify the success of the selective deposition on PDMS stamps, we replicated the polymer structures using capillary force lithography. ${ }^{18}$ Here, we used SBS as it has a relatively low glass transition temperature $\left(\sim 36{ }^{\circ} \mathrm{C}\right) .{ }^{18}$ After metal removal, the stamp makes conformal contact with the SBS surface as visually examined. However, a PDMS stamp with a very thin iron film (less than $5 \mathrm{~nm}$ ) can also make conformal contact under a slight pressure $\left(\sim 1 \mathrm{~N} / \mathrm{cm}^{2}\right)$ without metal removal. After annealing for a period of time, typically for $5 \mathrm{~h}$ at $80^{\circ} \mathrm{C}$, the patterned microstructures were examined by SEM. The inset shows the corresponding SBS structures fabricated by the three different stamps shown in Fig. 2. As shown in the figure, well-defined polymer replicas can be realized with high fidelity. In particular, the depth of the channel has not been changed in Fig. 2(c) as opposed to the AFM measurement, which indicates that the substantial decrease in step height indeed results from the tip effects, not from the failure in selectivity. The aspect ratios of the three structures are measured to be $1.4,1.9$, and 5 , respectively, corresponding to the reduced channel widths. The deposition rate along the sidewall appears not to be uniform during the polymerization process such that a slight increase in base width is observed as in Figs. 2(b) and 2(c). To gain a better edge definition, this phenomenon needs to be further addressed.

The technical usefulness of the modified stamp was further tested using $\mu \mathrm{CP}$ as shown in Figs. 2(d) and 2(e). To ensure conformal contact, no pressure was applied on top of the stamp throughout the contact process. As seen from the figures, well-defined $\mathrm{Au}$ lines were fabricated with feature sizes in complete agreement with those of the stamps used and etching resulted in complete removal of the $\mathrm{Au}$ and underlying Ti. ${ }^{19}$ These results suggest that conformable wetting can be maintained after deposition of Ti/Fe and then removal by HF.

In terms of pattern transfer, the stability and wettability of the modified PDMS stamp against heat and solvent need to be addressed. Figure 3 shows the change of a static contact angle for the stamp at the water/air interface at room temperature as determined by the presence of metal layer. Also shown in the figure are the effects of solvent or heat treatment on wetting conditions of the stamp after metal removal. A conformable wetting is restored after dipping in an acidic solution as judged by the contact angle change $\left(106^{\circ}\right.$ $\pm 1.5 \rightarrow 103^{\circ} \pm 3$ ), which agrees with the results in Fig. 2 . Furthermore, the contact angle did not change considerably with additional application of ethanol for $6 \mathrm{~h}$ or heat treat-

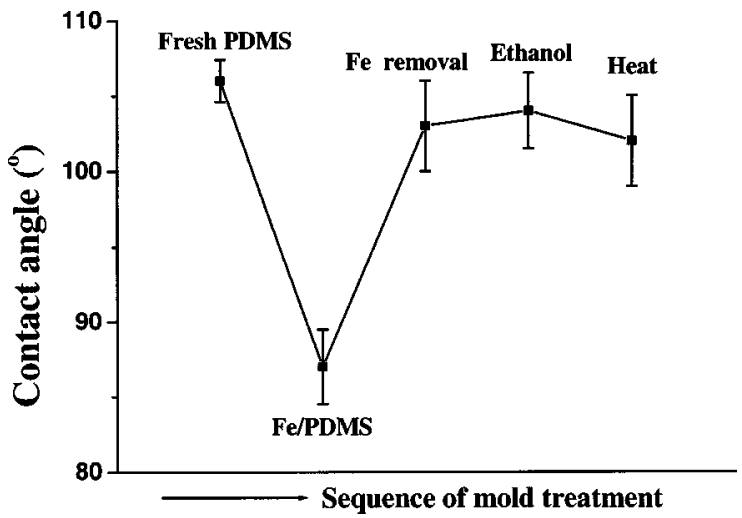

FIG. 3. Change of a static contact angle for the stamp at the water/air interface at room temperature as determined by the presence of metal layer and subsequent application of ethanol and heat treatment.

ment at $80^{\circ} \mathrm{C}$ for $6 \mathrm{~h}$, which indicates durability during molding or printing process at room temperature.

In summary, we have developed a method to fabricate polymer-reinforced PDMS stamps using chemical vapor deposition in combination with standard microfabrication steps. This convenient and flexible technology could enable an opportunity to fabricate unique microfluidics and bioMEMS devices if additional modifications are made using specific poly- $p$-xylylenes with active, functional groups.

The authors gratefully acknowledge the assistance of Gwen Donahue for electron-beam evaporation and scanning electron microscopy measurements. Work was performed, in part, in the MTL facility at MIT.

${ }^{1}$ Y. N. Xia and G. M. Whitesides, Annu. Rev. Mater. Sci. 28, 153 (1998).

${ }^{2}$ E. Delamarche, H. Schmid, B. Michel, and H. Biebuyck, Adv. Mater. (Weinheim, Ger.) 9, 741 (1997).

${ }^{3}$ A. Bietsch and B. Michel, J. Appl. Phys. 88, 4310 (2000).

${ }^{4}$ C. Y. Hui, A. Jagota, Y. Y. Lin, and E. J. Kramer, Langmuir 18, 1394 (2002).

${ }^{5} \mathrm{H}$. Schmid and B. Michel, Macromolecules 33, 3042 (2000).

${ }^{6}$ T. W. Odom, J. C. Love, D. B. Wolfe, K. E. Paul, and G. M. Whitesides, Langmuir 18, 5314 (2002).

${ }^{7}$ K. M. Choi and J. A. Rogers, J. Am. Chem. Soc. 125, 4060 (2003).

${ }^{8}$ G. Csucs, T. Künzler, K. Feldman, F. Robin, and N. D. Spencer, Langmuir 19, 6104 (2003).

${ }^{9}$ J. Lahann, D. Klee, and H. Höcker, Macromol. Rapid Commun. 19, 441 (1998).

${ }^{10}$ J. Lahann, I. S. Choi, J. Lee, K. F. Jensen, and R. Langer, Angew. Chem., Int. Ed. 40, 3166 (2001).

${ }^{11}$ J. Lahann, M. Balcells, T. Rodon, J. Lee, I. S. Choi, K. F. Jensen, and R. Langer, Langmuir 18, 3632 (2002).

${ }^{12}$ J. Lahann and R. Langer, Macromolecules 35, 4380 (2002).

${ }^{13}$ K. M. Vaeth and K. F. Jensen, Adv. Mater. (Weinheim, Ger.) 11, 814 (1999)

${ }^{14}$ Although the underlying mechanism is not fully understood, inhibition of polymer growth during CVD polymerization can also be observed if surfaces were previously exposed to aqueous solutions of iron salts [cf. K. M. Vaeth and K. F. Jensen, Adv. Mater. (Weinheim, Ger.) 11, 814 (1999)].

${ }^{15}$ A. Greiner, Trends Polym. Sci. 5, 12 (1997).

${ }^{16}$ P. Simon, S. Mang, A. Hasenhindl, W. Gronski, and A. Greiner, Macromolecules 31, 8775 (1998)

${ }^{17}$ C. Schmidt, V. Stumpflen, J. H. Wendorff, A. Hasenhindl, W. Gronski, M. Ishaque, and A. Greiner, Acta Polym. 49, 232 (1998).

${ }^{18}$ K. Y. Suh, Y. S. Kim, and H. H. Lee, Adv. Mater. (Weinheim, Ger.) 13, $1386(2001)$

${ }^{19}$ A. Kumar, H. A. Biebuyck, N. L. Abbott, and G. M. Whitesides, J. Am. Chem. Soc. 114, 9188 (1992) 